\title{
Management of Ovarian Cancer: Experience of a single Institution
}

\author{
Elkhouly. E. ${ }^{1}$, Abdelghany. A. ${ }^{1}$, Rageh. T. ${ }^{2}$ and Shehata. M. ${ }^{1}$ \\ ${ }^{1}$ Department of Clinical Oncology, ${ }^{2}$ Departement of General Surgery, Faculty of Medicine, Menoufia \\ University, Egypt.
}

\begin{abstract}
Objectives: Retrospective review of total management and survival analysis of epithelial ovarian cancer patients in a single institution.

Background: Epithelial ovarian cancer comprises the majority of ovarian neoplasms (about 80\%). Predisposing factors may be Genetic, personal history of breast or endometrial, nulliparity, endometriosis and postmenopausal estrogen. Symptoms are often vague and till now there are no effective screening programs. Typically, treatment depends on a combination of surgery and chemotherapy in most of the patients.

Methods: A retrospective review of medical files of all patients diagnosed and treated as epithelial ovarian cancer at clinical oncology department, Menoufia University from January 2006 till December 2011. The data collected included clinico-pathological characteristics, treatment modalities, response evaluation, progression free survival (PFS), and overall survival (OS).

Results: This study included 83 patients; the median age for patients was 55 years (18-72 years). $84.3 \%$ of the patients presented in advanced stages (Stages III and IV). Serous cystadenocarcinoma was the predominant pathologic subtypes in $68.7 \%$ of patients. Surgery was the initial treatment in $86.7 \%$ of the cases. All surgeries were done by Gyne-surgeons, Onco-surgeons, and General surgeons. Paclitaxel-carboplatin was the most commonly used regimen as first line chemo-therapy. Response rate to first line chemotherapy reached $80.2 \%$ (35\% complete response). The median PFS and OS after first line chemotherapy were 17 and 45 months respectively. None of the patients was involved in clinical trials.

Conclusion: In our study, the age incidence of ovarian cancer was 55 years. $84.3 \%$ of the patients' typical presentation was advanced stage disease. The PFS was nearly the same as reported in the western literature. There was a significant correlation between response and stage and the same for OS and PFS with the type of debulking.
\end{abstract}

Key words: Ovarian cancer, Epithelial subtype, Survival outcome

Corresponding Author: Enas Abou Baker Elkhouly

E-mail: enasaboubaker6@hotmail.com

\section{INTRODUCTION}

Epithelial ovarian cancer comprises the majority of ovarian neoplasms (about $80 \%)^{1}$. Ovarian cancer is the seventh most common cancer in women under the age of 65 years. A woman's risk of developing ovarian cancer by age 65 years ranges from $0.36 \%$ in developing countries to $0.64 \%$ in developed countries ${ }^{2}$. In Europe, just over a third of women with ovarian cancer live five years after diagnosis, largely because most women with ovarian cancer are diagnosed when the cancer is already at an advanced stage ${ }^{3}$.

Screening in post-menopausal women, show promise in identifying early-stage disease, although survival data are still pending ${ }^{4}$. Typically, treatment of epithelial ovarian cancer depends on a combination of surgery and chemotherapy in most of the patients, Improvement in surgical techniques and chemotherapy agents has resulted in a modest increase in the 5-years survival over the last three decades from $37 \%$ to $45 \%$, although, even now, two thirds of women die from their disease 5 .

In early-stage disease (Federation of International Gynecologists and Obstetricians (FIGO) stage I/II) radical surgery will cure most women, although a minority of women will benefit from adjuvant chemotherapy, especially those who are not adequately staged at primary surgery ${ }^{6}$. Unfortunately, around $75 \%$ of women present when the disease has spread outside the pelvis (FIGO stage III/IV), when surgery alone cannot be curative. The standard treatment at these advanced stages is staging laparotomy with primary debulking surgery (PDS) followed by platinum-based chemotherapy. The extent of tumor cytoreduction is considered the most important independent prognostic factor ${ }^{7}$. Surgery is recommended 


Vol. 10 | No. 1-2 $2014 \quad$ Elkhouly. E. et al.

to be performed by a specialist gynecologic oncologist surgeon in cases highly suspicious for malignancy ${ }^{8}$.

This is a retrospective study to analyze clinicpathological features, different treatment modalities (chemotherapy protocols, type of surgery), and treatment outcome of epithelial ovarian cancer patients.

\section{MATERIALS AND METHODS}

This is a retrospective study included 83 patients diagnosed as epithelial ovarian cancer presented to Menoufia University, clinical oncology department from January 2006 till December 2011 and followed up till June 2013. Data was collected from the available files of patients regarding: patients' characteristics (age and median age group), disease characteristics including pathology, grade, staging was based on TNM staging according to the American Joint Committee on Cancer (AJCC 2010). Treatment modalities offered to the patients including surgery and chemotherapy. Data collected regarding surgery includes: Type of surgeon, debulking surgery (optimal versus suboptimal), Time of debulking (initial, interval or delayed debulking). Regarding chemotherapy, in this study we focused on first line platinum based chemotherapy either single agent carboplatin or combination platinum based chemotherapy. Response to treatment was assessed according to RECIST version 1.1.

\section{Statistical Analysis:}

Data analysis using SPSS program for windows version 16 (SPSS ICN, Chicago II, USA). Tests used in analysis were Pearson Chi. Square test and fisher's exact test. They were used to determine the significance of associations between categorical variables and response. PFS was analyzed using the Kaplan. Meier curves. It was calculated from the date of diagnosis to the date of progression or the date of death (all causes), whichever occur first, patients who weren't progressed at last follow up were censored. Differences between groups were assessed by means of the log-rank test. Two-sided $P$-value $<0.05$ was considered statistically significant.

\section{RESULTS}

Clinico-pathologic criteria for the studied patients represented in table 1. Median age for all patients in the study was 55 years (18-72 years), patients are classified into two groups according to this median age, first group was $\geq 55$ years which were slightly more than half of the patients $(54.2 \%)$ while the other group was $<55$ years which were $(45.8 \%)$. Serous cystadenocarcinoma was the prominent histological type $(68.7 \%)$, followed by endometrioid type $(18.1 \%)$ and lastly mucinous type (13.3\%). Most of patients had high grade tumors (74.7\%),
$19.3 \%$ are grade I, and grade II represented $6 \%$ of the patients. Advanced disease (stage III and IV) presented in $84.3 \%$ of the patients, while $15.7 \%$ of patients were stage I and II.

Analysis of treatment is shown Table 2. Seventy two patients $(86.7 \%)$ underwent debulking surgery and eleven patients (13.3\%) didn't. Early debulking was done for $59 \%$ of cases. Fifty three percent of patients underwent optimal debulking while $47 \%$ had suboptimal debulking. Oncosurgeon performed $38.9 \%$ of surgery, gynecologist did $34.7 \%$, and general surgeon did $26.4 \%$. All patients received chemotherapy. 77 patients completed six cycles while 6 patients died before treatment was completed. Combination platinum based chemotherapy was received by $86.7 \%$ of patients, while only $13.3 \%$ received single agent carboplatin (Table 2).

Forty patients were eligible for assessment of response. After first line platinum based chemotherapy, $35 \%$ of patients had complete response (CR), while $55 \%$ had partial response (PR), $2.5 \%$ had stable disease (SD), and $7.5 \%$ had disease progression (DP) (Table 3). Correlation between response and stage is shown in Table 4.

Median follow up was 31 months with $67.5 \%$ of patients progressed during follow up while 27 patients $(32.5 \%)$ remained progression free. Fifty one patients were alive while 32 cases died during the follow up period (table 5).

Median PFS was 17 month while the median OS was 45 months. Kaplan Meier curve shows a statistically significant correlation between PFS and stage $(P$. value $=$ 0.001) (Figure1). There is a statistically significant correlation between PFS in months and type of debulking surgery $(P$. value $=0.036)$ (Figure 2$)$. Correlation between OS in months and debulking surgery was statistically significant $(P$. value $=0.003)$ (Figure 3). Kaplan Meier curve shows a statistically significant correlation between OS in months and chemotherapy regimen $(P$. value $=$ 0.007) (Figure 4).

PFS univariate analysis, stage and type of debulking were statistically significant, but after multivariate analysis by Cox regression, stage was independent prognostic factor with significance of $P=0.008$, hazard ratio $=4.228,95 \%$ confidence interval $(\mathrm{CI})=1.448$ 12.341, while type of debulking lost significance.

Univariate analysis of OS, type of debulking and chemotherapy regimen were statistically significant, after multivariate analysis by Cox regression, type of debulking was independent prognostic factor with significance of $P=0.003$, hazard ratio (HR) $=2.977,95 \%$ 
$\mathrm{CI}=1.435-6.173$, and similarly chemotherapy regimen was independent prognostic factor with significance of $P=0.005, \mathrm{HR}=0.296,95 \% \mathrm{CI}=0.125-0.699$

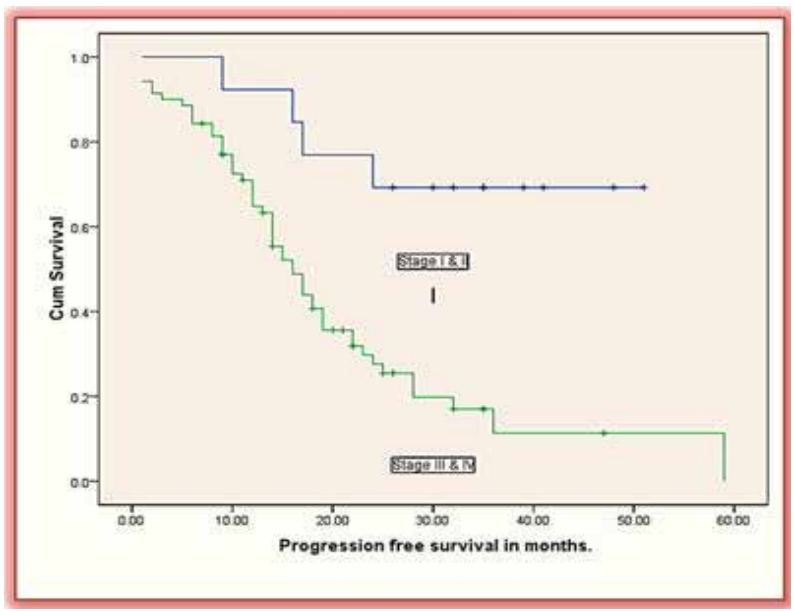

Figure 1: Kaplan Meier curve shows association of PFS and stage $(P=0.001)$.

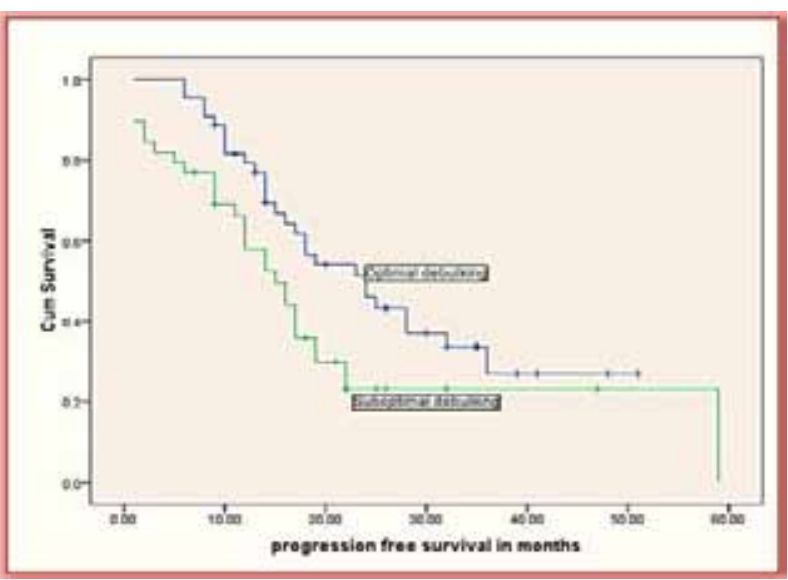

Figure 2: Kaplan Meier curve shows association of PFS and type of debulking $(P=0.036)$.

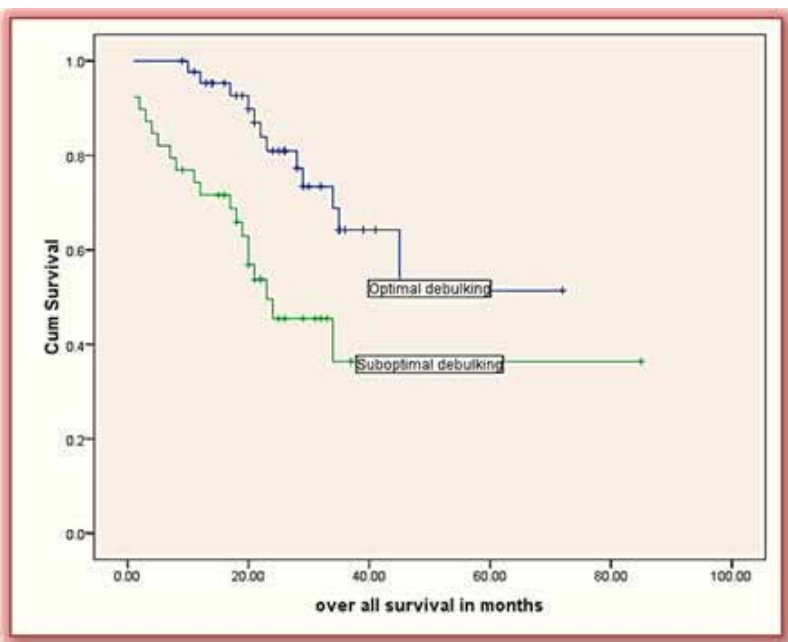

Figure 3: Kaplan Meier curve shows association of OS and type of debulking $(P=0.003)$.

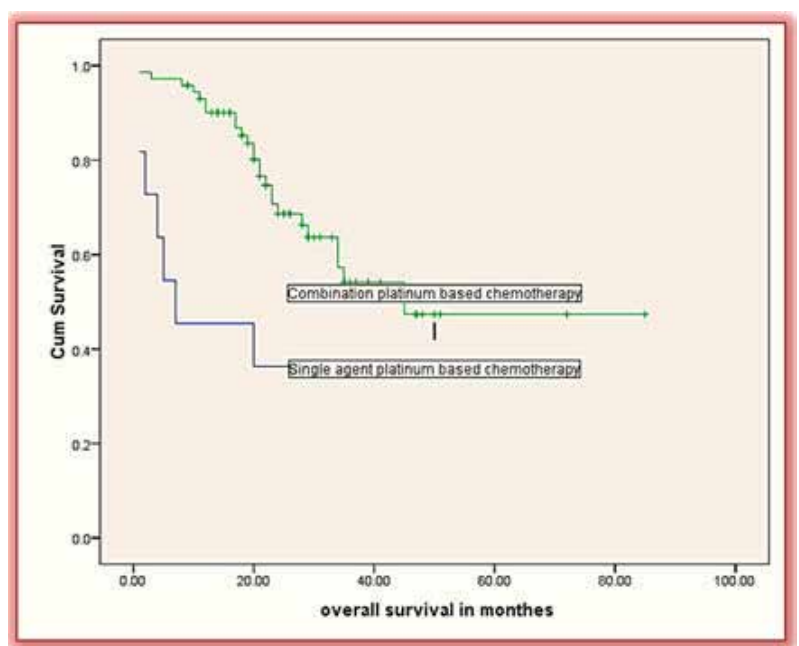

Figure 4: Kaplan Meier curve shows association of OS and chemotherapy regimen $(P=0.007)$.

Table 1: Patients clinico-pathological characteristics.

\begin{tabular}{|c|c|c|c|}
\hline Characteristics & & No of patients & $\%$ \\
\hline \multirow{2}{*}{ Age } & $<55$ & 38 & 45.8 \\
\hline & $\geq 55$ & 45 & 54.2 \\
\hline \multirow{4}{*}{ Type of biopsy } & Histology & 54 & 65.1 \\
\hline & Cytology & 6 & 7.2 \\
\hline & Cytology and postchemotherapy histology & 10 & 12 \\
\hline & Histology and postchemotherapy histology & 13 & 15.7 \\
\hline \multirow{3}{*}{ Grade } & Grade I & 16 & 19.3 \\
\hline & Grade II & 5 & 6 \\
\hline & Grade III & 62 & 74.7 \\
\hline \multirow{3}{*}{ Pathology } & Serous & 57 & 68.7 \\
\hline & Mucinous & 11 & 13.3 \\
\hline & Endometrioid & 15 & 18.1 \\
\hline \multirow{4}{*}{ Stage } & Stage I & 11 & 13.3 \\
\hline & Stage II & 2 & 2.4 \\
\hline & Stage III & 49 & 59 \\
\hline & Stage IV & 21 & 25.3 \\
\hline
\end{tabular}


Table 2: Analysis of the treatment received by the patients

\begin{tabular}{|c|c|c|c|c|}
\hline Treatment analysis & No of patients & Percent & No of patients & $\%$ \\
\hline \multirow{9}{*}{ surgery } & \multirow{4}{*}{ Timing of surgery } & Early debulking & 49 & $59 \%$ \\
\hline & & Interval debulking & 14 & $16.9 \%$ \\
\hline & & Delayed debulking & 9 & $10.8 \%$ \\
\hline & & Only Biopsy & 11 & $13.3 \%$ \\
\hline & \multirow{2}{*}{ Type of debulking } & Optimal & 44 & $53 \%$ \\
\hline & & Suboptimal & 39 & $47 \%$ \\
\hline & \multirow{3}{*}{ Type of surgeon } & General surgeon & 19 & $26.4 \%$ \\
\hline & & Oncosurgeon & 28 & $38.9 \%$ \\
\hline & & Gynecologist & 25 & $34.7 \%$ \\
\hline \multirow{7}{*}{ chemotherapy } & \multirow{2}{*}{ Timing of chemotherapy } & Neoadjuvant & 30 & $36.1 \%$ \\
\hline & & Adjuvant & 53 & $63.9 \%$ \\
\hline & \multirow{2}{*}{ Chemotherapy regimen } & Single agent carboplatin & 11 & $13.3 \%$ \\
\hline & & Combination carboplatin based & 72 & $86.7 \%$ \\
\hline & \multirow{3}{*}{ Platinum sensativety } & Platinum sensative & 62 & $74.7 \%$ \\
\hline & & Platinum resistant & 15 & $18.1 \%$ \\
\hline & & Not assessed & 6 & $7.2 \%$ \\
\hline
\end{tabular}

Table 3: Treatment response of the patients

\begin{tabular}{lcc}
\hline Response & No of patients & percent \\
\hline Complete response & 14 & $35 \%$ \\
\hline Partial response & 22 & $55 \%$ \\
\hline Stable disease & 1 & $2.5 \%$ \\
\hline Disease progression & 3 & $7.5 \%$ \\
\hline Total & 40 & $100 \%$ \\
\hline
\end{tabular}

Table 4: Relation between treatment response and stage.

\begin{tabular}{|c|c|c|c|c|}
\hline \multirow{2}{*}{$\begin{array}{l}\text { Complete } \\
\text { response }\end{array}$} & \multicolumn{2}{|c|}{ Stage } & \multirow[b]{2}{*}{ total } & \multirow{2}{*}{$P$. value } \\
\hline & Stage III & Stage IV & & \\
\hline \multirow{2}{*}{ Yes } & 12 & 2 & 14 & \\
\hline & $52.2 \%$ & $11.8 \%$ & $35 \%$ & \\
\hline \multirow{2}{*}{ No } & 11 & 15 & 26 & \\
\hline & $47.8 \%$ & $88.2 \%$ & $65 \%$ & \\
\hline \multirow{2}{*}{ Total } & 23 & 17 & 40 & \\
\hline & $100 \%$ & $100 \%$ & $100 \%$ & 0.009 \\
\hline
\end{tabular}

Table 5: Analysis of disease progression and living status at June 2013 (median follow up 31 months).

\begin{tabular}{|c|c|c|c|}
\hline Status & & $\begin{array}{c}\text { No of } \\
\text { patients }\end{array}$ & percen \\
\hline \multirow{2}{*}{ Progression status } & Progression free & 27 & $32.5 \%$ \\
\hline & Progressed & 56 & $67.5 \%$ \\
\hline \multirow{2}{*}{ Living status } & Living & 51 & $61.4 \%$ \\
\hline & Dead & 32 & $38.6 \%$ \\
\hline
\end{tabular}

\section{DISCUSSION}

Ovarian cancer incidence is strongly related to age, with the highest incidence rates being in older women. In our study we find that the mean age was 53.4 years with a range of 18-72 years and median age was 55 years. In the Gharbia population based cancer registry, the mean age at diagnosis was 47.2 years and the median age was 49 years ${ }^{9}$.

In UK between 2008 and 2010, an average of 53\% of cases diagnosed in women aged 65 years ${ }^{10}$. In USA, the median age at the time of diagnosis is 63 years $^{10}$. So, the Age of ovarian cancer incidence in our study is 10 years younger than seen in UK \& USA. This is could be explained by difference in sample size, age at menarche, or other reasons that worth further confirmation studies.

Among our patients, $68.7 \%$ were serous carcinomas, $13.3 \%$ were mucinous carcinomas and $18.1 \%$ were endometrioid. In the Middle East consortium study, serous carcinomas predominated with percentage ranging between $27.2 \%$ and $49.9 \%$, followed by adenocarcinomas. The proportion of mucinous carcinomas among Egyptians in this study was 16.1\% and among Jordanians was $11.7 \%$ whereas in Israeli and Cyprious registries the percentage was lower ranging from $6 \%$ to $8.7 \%{ }^{11}$.

Paes, et al. ${ }^{10}$, found that $30 \%$ of the epithelial tumors were serous while $13.7 \%$ were mucinous. So, the incidence of serous among all ovarian cancer cases in our study is higher than those reported by others, while mucinous is nearly the same. This difference could be 
Kasr-El-Aini Journal Of Clinical Oncology And Nuclear Medicine

Vol. 10 |No. 1-2 2014

explained by a relative a relative small sample size of the present study or predominance of molecular phenotype and genotype expressing more serous histology.

The majority of patients presented in late stages III and IV (84.3\% of the cases). Our results were the highest among most of other studies. In Pakistan, $\mathrm{Pak}^{12}$ found that $78 \%$ of the cases were in stages III and IV at presentation. Paes, et $a l .{ }^{10}$ in their study found that stages III and IV accounted for only $56.2 \%$ of his cases. This can be explained by low social class of patients in Menoufia.

In the present study $86.7 \%$ of patients were treated initially with surgery. In a study evaluating ovarian cancer in oriental women from Singapore found that surgery was the primary treatment modality in $97 \%$ of the cases $^{13}$. In a university hospital in Berlin that reviewed 372 consecutive patients with advanced ovarian cancer found that $89 \%$ of the cases underwent surgery ${ }^{14}$.

In the Medicare population evaluating patients with advanced ovarian epithelial carcinoma found that surgery was performed initially in $58.8 \%$ of the women which is much lower than our study, German and the Singapore trials ${ }^{15}$.

Of those who underwent surgery 53\% had optimum cytoreduction surgery (no residual or residual less than $2 \mathrm{~cm}$ ). In the study evaluating surgery in 115 patients with stages III and IV ovarian cancer in the south west of the Netherlands, the investigators found that optimal surgery was done in only $45 \%$ of the cases ${ }^{16}$. Survey study among gynecologist performing surgery for ovarian carcinoma in Australia and New Zealand, they found that about $65 \%$ of the surgeons perform optimal cytoreductive surgery ${ }^{17}$. It is obvious from the comparison that in our series, optimal cytoreductive surgery is performed in the median range between two previously mentioned trials and this can be explained by; the experience of our surgeons and their awareness of their surgical fields as $73.6 \%$ of optimal debulking done by gynecologists or oncosurgeons.

In the present study, all patients received platinum based chemotherapy, 77 patients completed six cycles chemotherapy and 6 patients received only one or two cycles and died. Thirty six percent of the cases started neoadjuvant chemotherapy. As regard the type of chemotherapy regimen, Paclitaxel-carboplatin was the most frequently used regimen as first line in $86.7 \%$ of the cases and single agent carboplatin was used in $13.3 \%$ of the cases (two cases indicated for only single agent carboplatin and others used it alone for fragile patients with moderate performance status or when there is shortage in paclitaxel because of the limited resources).

All of our cases were sub-optimally debulked stage III or stage IV either with initial, interval or delayed debulking. The response rate to the first line chemotherapy after six cycles of chemotherapy was seen in $90 \%$ of the cases (CR 35\%), if we add cases with stable disease after three cycles chemotherapy, the overall response will increase to $92.5 \%$. Only $7.5 \%$ of the cases progressed after six cycles of first line chemotherapy. There are different percentages of complete responses ${ }^{18}$. In the GOG study protocol 47, they found that the complete response rate for the cisplatin containing arm reached $51 \%$. The response rate to paclitaxel followed by either cisplatin or carboplatin in the exploratory phase III study ranged between (64-74\%) $)^{19}$, while the pathological complete response in another Phase III trial comparing paclitaxel plus cisplatin versus paclitaxel plus carboplatin, was nearly similar among both groups (46 vs. $53 \%)^{20}$.

The possible explanation of the difference in clinical complete response between our study and the different international studies could be explained by: first, the number of the patients in our study was relatively small in comparison to those studies. Second, no standard chemotherapy protocol was given among all patients in our study (most of patients received single agent carboplatin were indicated for combination paclitaxel and carboplatin), and third: the high frequency of chemotherapy underdosage and frequent interruption of the treatment were due to limited resources and unavailability of the drugs specially paclitaxel (i.e. lower compliance).

In the present study, PFS after first line chemotherapy was 17 months which is largely similar to the results of international studies ${ }^{18-22}$.

Poorer results were associated with advanced clinical stage (III \& IV), suboptimal surgery or no surgery at all. Differences were statistically significant. These results agree with reported series by other investigators.

Higher tumor grade had lower PFS compared to low grade tumor, 20.5 versus 34.5 months. The difference was statistically significant $(P=0.002)$.

There was a statistically significant correlation between OS and age $(P$. value $=0.039)$. The median OS for patients of age group ( $<55$ years) was 60 months while for older patients ( $\geq 55$ years) was 37.7 months. This is could be explained by poor tolerance of elderly especially who presented by advanced disease to standard combination chemotherapy, associated co-morbidities which made the patient inoperable, and patient had bulky disease also increases the risk of death from other causes.

\section{CONCLUSION}

The age incidence of ovarian cancer in our study is fifty five years which is eight years higher than that 
of other parts of Egypt. For all patients in this study, typical presentation was late. The response rate to first line chemotherapy was high and the PFS was nearly the same as reported in the western literature. The response was significantly correlated to stage. There was significant correlation for PFS with the grade, stage, and debulking surgery. Also OS significantly correlated to median age group, type of debulking, and chemotherapy regimen. The main limitation of the study is the small sample size, low compliance to treatment, suboptimal treatment, and short follow up.

\section{Disclosure}

The authors declare no conflict of interest

\section{REFERENCES}

1. Chan JK, Cheung MK, Husain A, Teng NN, West D, Whittemore AS, et al. Patterns and progress in ovarian cancer over 14 years. Obstet.Gynecol. 2006; 108(3 I):521-8.

2. Ferlay J, Shin HR and Bray F. GLOBOCAN 2008: Cancer Incidence and Mortality Worldwide.2010 IARC Cancer Base No. 10. International Agency for Research on Cancer, Lyon, France.

3. Jemal A, Siegel Rb, Ward E, Hao Y, Xu J, Murray T, et al. Cancer statistics, 2008. CA Cancer J.Clin. 2008;58(2):71-96.

4. Menon U, Gentry Maharaj A, Hallett R, Ryan A, Burnell M, Sharma A, et al. Sensitivity and specificity of multimodal and ultrasound screening for ovarian cancer and stage distribution of detected cancers: Results of the prevalence screen of the UK Collaborative Trial of Ovarian Cancer Screening (UKCTOCS). Lancet Oncol. 2009;10(4):327-40.

5. Jemal A, Siegel R, Ward E, Hao Y, Xu J, Thun MJ. Cancer statistics, 2009. CA Cancer J Clin. 2009 JulAug;59(4):225-49.

6. Trimbos B, Timmers P, Pecorelli S, Coens C, Ven K, van der Burg M, et al. Surgical staging and treatment of early ovarian cancer: Long-term analysis from a randomized trial. J Natl Cancer Inst. 2010 Jul 7;102(13):982-7.

7. Vergote I, Tropé CG, Amant F, Kristensen GB, Ehlen T, Johnson $\mathrm{N}$, et al. Neoadjuvant chemotherapy or primary surgery in stage IIIC or IV ovarian cancer. N Engl J Med. 2010;263(10):943-53.

8. Engelen MJ, Kos HE, Willemse PH, Aalders JG, de Vries EG, Schaapveld M, et al. Surgery by consultant gynecologic oncologists improves survival in patients with ovarian carcinoma. Cancer. 2006 ;106(3):589-98.

9. Abdel bar I. Cancer in Egypt, Gharbiah. Triennial report of 2000 2002. 1st ed.: Gharbiah population based cancer registry; 2007.
10. Barakat R, Berchuck A, Markman M. Principles and practice of gynecologic oncology. 6th ed. Philadelphia: Lippincott Williams \& Wilkins; 2013.

11. Freedman LS, Al Kayed S, Qasem MB, Barchana M, Boyiadzis M, El Najjar K, et al. Cancer registration in the Middle East. Epidemiology. $2001 \mathrm{Jan} ; 12(1): 131-3$.

12. Malik IA. A prospective study of clinco-pathological features of epithelial ovarian cancer in Pakistan. J Pak Med Assoc. 2002;52:155-8.

13. Singh P, Arunachalam I, Singh P, Tan BY, Tock EP, Ratnam SS. Ovarian cancer in Oriental women from Singapore: Disease pattern and survival. Int Surg. 1990;75(2):115-22.

14. Sehouli J, Savvatis K, Braicu EI, Schmidt SC, Lichtenegger W, Fotopoulou C. Primary versus interval debulking surgery in advanced ovarian cancer: Results from a systematic single-center analysis. Int J Gynecol Cancer 2010 Nov;20(8):1331-40.

15. Thrall MM, Gray HJ, Symons RG, Weiss NS, Flum DR, Goff BA. Trends in treatment of advanced epithelial ovarian cancer in the Medicare population. Gynecol Oncol. 2011 Jul;122(1):100-6.

16. Gerestein CG, Eijkemans MJ, Bakker J, Elgersma OE, van der Burg ME, Kooi GS, et al. Nomogram for suboptimal cytoreduction at primary surgery for advanced stage ovarian cancer. Anticancer Res. 2011 Nov;31(11):4043-9.

17. Brand AH. Ovarian cancer debulking surgery: A survey of practice in Australia and New Zealand. Int J Gynecol Cancer 2011 Feb;21(2):230-5.

18. Thigpen T, Vance R, Puneky L, Khansur T. Chemotherapy in advanced ovarian carcinoma: Current standards of care based on randomized trials. Gynecol Oncol. 1994 Dec;55(3 Pt 2):S97-S107.

19. Neijt JP, Engelholm SA, Tuxen MK, Sorensen PG, Hansen $\mathrm{M}$, Sessa C, et al. Exploratory phase III study of paclitaxel and cisplatin versus paclitaxel and carboplatin in advanced ovarian cancer. J Clin Oncol. 2000 Sep;18(17):3084-92.

20. Ozols RF, Bundy BN, Greer BE, Fowler JM, Clarke Pearson D, Burger RA, et al. Phase III trial of carboplatin and paclitaxel compared with cisplatin and paclitaxel in patients with optimally resected stage III ovarian cancer: A Gynecologic Oncology Group study. J Clin Oncol. 2003 Sep;21(17):3194-200.

21. McGuire WP, Hoskins WJ, Brady MF, Kucera PR, Partridge EE, Look KY, et al. Cyclophosphamide and cisplatin compared with paclitaxel and cisplatin in patients with stage III and stage IV ovarian cancer. N Engl J Med. 1996 Jan;334(1):1-6.

22. Muggia FM, Braly PS, Brady MF, Sutton G, Niemann TH, Lentz SL, et al. Phase III randomized study of cisplatin versus paclitaxel versus cisplatin and paclitaxel in patients with suboptimal stage III or IV ovarian cancer: A gynecologic oncology group study. J Clin Oncol. 2000 Jan;18(1):106-15. 